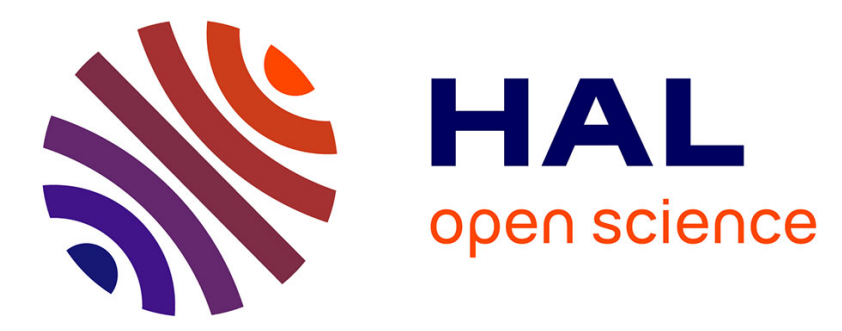

\title{
Generalized PEEC Analysis of Inductive Coupling Phenomena in a Transmission Line Right-of-Way
}

L. Blattner Martinho, J. Siau, B. Bannwarth, J. -M. Guichon, Olivier

Chadebec, Gérard Meunier, V. C. Silva

\section{- To cite this version:}

L. Blattner Martinho, J. Siau, B. Bannwarth, J. -M. Guichon, Olivier Chadebec, et al.. Generalized PEEC Analysis of Inductive Coupling Phenomena in a Transmission Line Right-of-Way. IEEE Transactions on Magnetics, 2016, 52 (3), 10.1109/TMAG.2015.2470249 . hal-02277769

\section{HAL Id: hal-02277769 \\ https://hal.science/hal-02277769}

Submitted on 23 Nov 2020

HAL is a multi-disciplinary open access archive for the deposit and dissemination of scientific research documents, whether they are published or not. The documents may come from teaching and research institutions in France or abroad, or from public or private research centers.
L'archive ouverte pluridisciplinaire HAL, est destinée au dépôt et à la diffusion de documents scientifiques de niveau recherche, publiés ou non, émanant des établissements d'enseignement et de recherche français ou étrangers, des laboratoires publics ou privés. 


\title{
Generalized PEEC Analysis of Inductive Coupling Phenomena in a Transmission Line Right-of-Way
}

\author{
L. Blattner Martinho ${ }^{1,2,3}$, J. Siau ${ }^{2,3,4}$, B. Bannwarth ${ }^{2,3}$, J.-M. Guichon ${ }^{2,3}$, O. Chadebec $^{2,3}$, G. Meunier ${ }^{2,3}$, V. C. Silva ${ }^{1}$ \\ ${ }^{1}$ Escola Politécnica da Universidade de São Paulo, LMAG, 05508-010 São Paulo, Brazil \\ ${ }^{2}$ Université Grenoble Alpes, G2Elab, F-38000 Grenoble, France \\ ${ }^{3}$ CNRS, G2Elab, F-38000 Grenoble, France \\ ${ }^{4}$ CEDRAT, F-38240 Meylan, France
}

\begin{abstract}
Two variations of a 3-D inductive coupling problem in a transmission line right-of-way are analysed with the generalized Partial Element Equivalent Circuit (PEEC) method. This new approach does not rely on the parallelism with the transmission line and permits the determination of the induced current density in underground objects at arbitrary positions and orientations. The adaptions on the basic numerical procedure are presented and discussed as well. A comparison of the obtained results with 2-D and 3-D Finite Element models shows that the proposed approach is able to provide accurate solutions even when employing a coarser discretization mesh.
\end{abstract}

Index Terms-Eddy Currents, electromagnetic coupling, generalized partial element equivalent circuit (PEEC) method, integral equation, transmission lines.

\section{INTRODUCTION}

$\mathbf{T}$ HE inductive coupling between high voltage transmission lines and other structures sharing a common right-ofway is unavoidable. The numerical techniques used to model this class of problems tend to take advantage whenever it is possible of the parallelism between the transmission line and elongated structures [1] [2]. For instance, this is the case of buried pipelines or transportation rails placed in a right-of-way and subjected to the influence of a transmission line.

Among the techniques employed in this context of applications is the Finite Element Method (FEM). In those situations involving the parallel alignment of long structures with the transmission line, the shared right-of-way may be reduced to one of its cross-sections and 2-D implementations of this method may be employed [2]. Such an approach leads to numerical problems of reasonable size and complexity, even if large inactive air regions and thin phase conductors need to be meshed.

However, the general inductive coupling problem is given by an object of arbitrary shape and with an arbitrary relative position with respect to the transmission line. Hence, the modelling of this broader class of situations cannot rely on the parallelism between structures as a simplification. As a consequence, larger and costlier numerical problems are obtained. The 3-D FEM may be applied to this general problem as well.

This paper proposes an alternative approach based on an integral method that also uses a complete 3-D representation of the right-of-way. More specifically, an adapted version of the generalized Partial Element Equivalent Circuit (PEEC) integral method will be presented and employed to determine the induced current density in an object beneath the soil surface and in the vicinities of a three-phase transmission line.

Two variants of the problem will be considered; the first is reducible to a 2-D model, while the second is not. According with the case, the results obtained with the PEEC procedure will be compared either to a 2-D or to a 3-D FEM counterpart solution, as will be discussed in the following sections.

\section{Generalized PEEC Method}

The generalized PEEC approach arises from an application of the Galerkin Residual method to the integral equation

$$
\frac{\mathbf{J}}{\sigma}+j \omega \frac{\mu_{0}}{4 \pi} \int_{\Omega} \frac{\mathbf{J}}{r} \mathrm{~d} \Omega=-\nabla V,
$$

which states the frequency domain relationship between the current density $\mathbf{J}$ and the electric potential $V$ in a three dimensional domain $\Omega$ [3]. The media in $\Omega$ are supposed to be non-magnetic, non-dielectric and with conductivity $\sigma$.

The procedure requires a finite element approximation for $\mathbf{J}$ with the use of vector facet shape functions $\left\{\mathbf{w}_{i}\right\}$ [4]. It leads to the assembly of the terms

$$
\begin{aligned}
R_{i j} & =\int_{\Omega} \frac{\mathbf{w}_{i} \cdot \mathbf{w}_{j}}{\sigma} \mathrm{d} \Omega, \\
L_{i j} & =\frac{\mu_{0}}{4 \pi} \int_{\Omega} \mathbf{w}_{i} \cdot\left(\int_{\Omega} \frac{\mathbf{w}_{j}}{r} \mathrm{~d} \Omega\right) \mathrm{d} \Omega \text { and } \\
\Delta V_{i} & =-\int_{\Omega} \mathbf{w}_{i} \cdot \nabla V \mathrm{~d} \Omega
\end{aligned}
$$

into a global system of equations with the following structure:

$$
([R]+j \omega[L])\left[I_{f}\right]=[\Delta V] .
$$

This system may be regarded as an equivalent electric circuit, in which each branch corresponds to a facet of the finite 
element mesh adopted for the discretization of $\mathbf{J}$. Matrices $[R]$ and $[L]$ are its equivalent resistance and inductance matrices and vector $\left[I_{f}\right]$ stores facet (or branch) currents. Additionally, it may be shown that the right-hand side vector $[\Delta V]$ contains the differences between the averaged electric potential of two contiguous elements sharing a common facet [3].

As a consequence of this circuit interpretation, the problem given by (3) may be coupled to a complementary external network and analyzed with an electric circuit solver. The solution with a circuit solver and the use of facet elements assure together the conservation of current, which is equivalent to the satisfaction of the required physical condition given by the continuity equation $\nabla \cdot \mathbf{J}=0$ [3].

It must be noticed that the circuit relations in (3) are stated in terms of unknown branch currents $\left[I_{f}\right]$. However, it is a well-known fact that circuit solvers are normally conceived to employ independent loop currents or nodal tensions as unknowns instead of branch currents. In this way, an independent loop search algorithm is required to convert the problem given by (3) into a well-posed form for mesh current analysis [5]. An inverse transformation from independent loop currents to branch currents is required as well after the solution of the equivalent electric circuit, in order to proceed with the evaluation of field quantities during the post-processing stage of the analysis.

The following sections will describe the inductive coupling problem of application considered and the required adaptions on the basic generalized PEEC method. A discussion on the obtained results will also be provided.

\section{Analysis of Inductive Coupling Problems in A RIGHT-OF-WAY}

A schematic view of the right-of-way under analysis is available in Fig. 1. The buried object under investigation has a prismatic and elongated shape and is much more conductive than the surrounding soil, which is supposed to have a uniform and isotropic resistivity.

As mentioned before, the computation of the current density distribution $\mathbf{J}$ induced by the high-voltage transmission line in the buried object requires adaptations in the numerical scheme outlined in section II. These modifications are covered in the following subsections, together with additional relevant information about the numerical modelling of this particular problem.

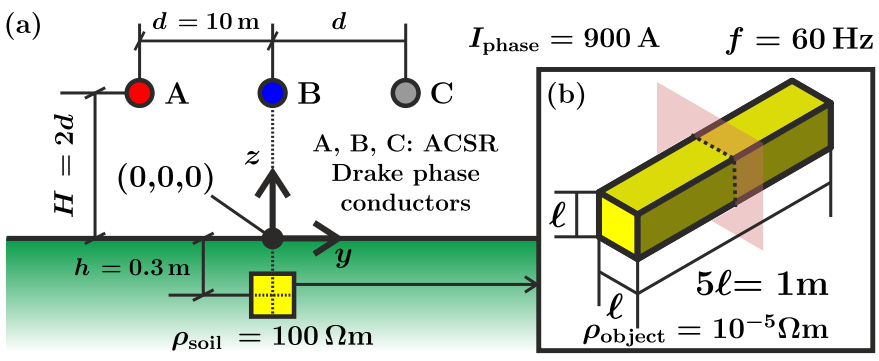

Fig. 1. The right-of-way (a) and the conductive object underground (b).

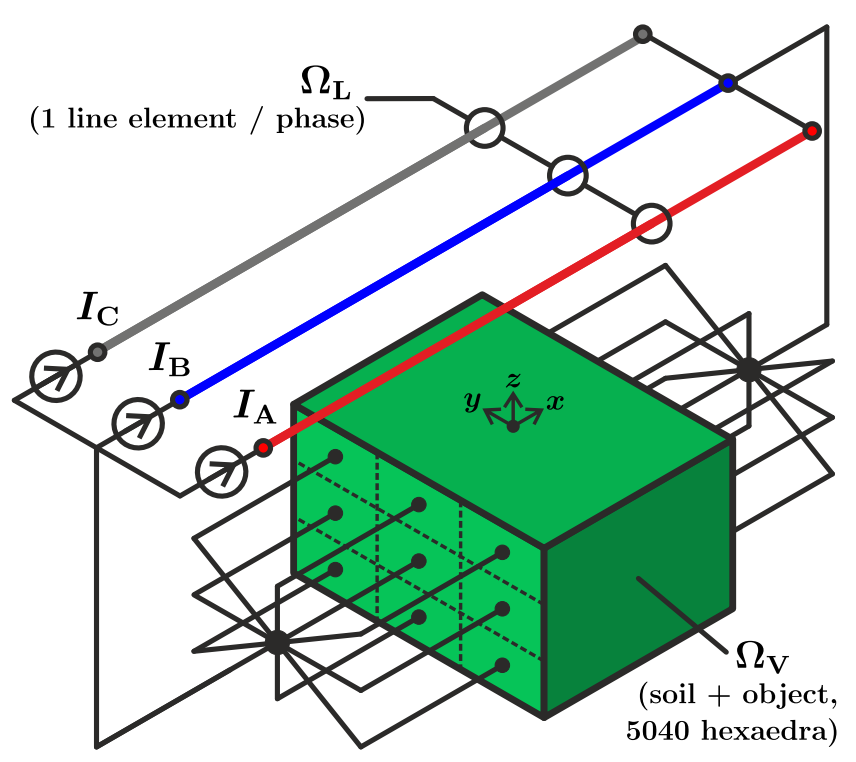

Fig. 2. The computational domain and its connections to the external circuit.

\section{A. Three-phase line representation and external circuit}

Fig. 2 shows the delimitation of the domain $\Omega$ and its decomposition into two sub-regions. A sufficiently large soil volume $\Omega_{\mathrm{V}}$ bounded by the earth surface and containing the embedded underground object is defined. In $\Omega_{\mathrm{V}}, \mathbf{J}$ is approximated by vector facet elements [4] associated to the function space $\left\{\mathbf{v}_{i}\right\}$ of vector facet functions.

The transmission line $\Omega_{\mathrm{L}}$ is represented by line elements, in order to avoid the volume discretization of the very thin phase conductors and to limit the number of additional degrees of freedom. Each phase conductor is associated to a single, long line element, which is supposed to carry a constant complex current. This corresponds to the adoption of a space of zeroorder interpolation functions $\left\{\mathbf{u}_{i}\right\}$ with a pre-defined direction for the description of the current distribution in $\Omega_{\mathrm{L}}$.

Fig. 2 also shows the required external circuit connections to provide current excitation and to establish an underground path for the flow of zero-sequence current components (if an unbalanced operation condition was to be considered).

\section{B. Block integration and assembly of the system of equations}

The choice of two different function spaces for the approximation of the current density distribution in $\Omega=\Omega_{\mathrm{V}} \cup \Omega_{\mathrm{L}}$ may be formally regarded as a coupling between the generalized PEEC method (applied to the soil and the buried object) and the classical PEEC method (applied to the transmission line) [6] [7]. The terms assembled to the global system of equations are still given by (2), but with the additional remark that now $\left\{\mathbf{w}_{i}\right\}=\left\{\mathbf{v}_{i}\right\} \cup\left\{\mathbf{u}_{i}\right\}$. As a consequence, matrices $[R]$ and $[L]$ in (3) acquire a $2 \mathrm{X} 2$ block structure.

Matrix $[R]$ is sparse and its blocks are integrated and stored in a rather conventional way. On the other hand, and as suggested by $(2 b),[L]$ is densely populated. Since the assembly time of a dense matrix and the memory required for its storage tend to grow very quickly with the size of 
the numerical problem, each of the blocks of this equivalent inductance matrix is treated in a particular way.

The largest of these inductive blocks corresponds to the $\Omega_{\mathrm{V}} \times \Omega_{\mathrm{V}}$ interaction. In order to mitigate the difficulties previously mentioned, this block is compressed using the $\mathcal{H}$-matrix representation and is treated with the HCA technique [8].

The inductive block associated to the $\Omega_{\mathrm{L}} \times \Omega_{\mathrm{L}}$ interaction is a small $3 \mathrm{X} 3$ matrix that is equivalent to the inductance matrix of the three-phase line. Its terms could be computed by the numerical integration of (2b) with a Gaussian quadrature routine. However, these computations are particularly prone to errors linked to the evaluation of singular integrands. Alternatively, this block is substituted by analytical computations of the conductor's self-inductances and of the mutual inductances between phases [9].

The two remaining blocks correspond to the inductive coupling between $\Omega_{\mathrm{L}}$ and $\Omega_{\mathrm{V}}$. Only one of these blocks needs to be computed since the other may be obtained by matrix transposition.

\section{Application And Results}

The application of the PEEC scheme described in the previous sections was carried out for two variants of the problem of Fig. 1, each one given by a different relative position between the three-phase line and the object.

On both cases, structured meshes containing 5040 hexahedra were adopted for the discretization of $\Omega_{\mathrm{V}}$. Similarly, the complete equivalent network arising from the PEEC numerical scheme in each variant case had approximately 15000 branches and 9500 independent current loops.

The results were compared with FEM computations performed with the Flux software package [10]. A detailed discussion on the two problems analyzed is given in what follows.

\section{A. Parallel alignment and 2-D FEM validation}

First, the longest dimension of the object is placed in parallel to the line. This configuration permits the validation of

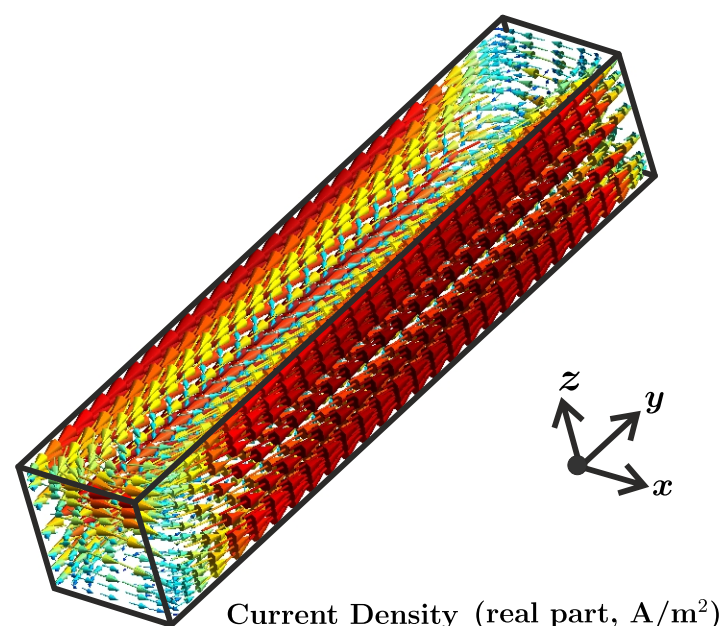

12.5

Fig. 3. Spatial distribution of the induced current density.

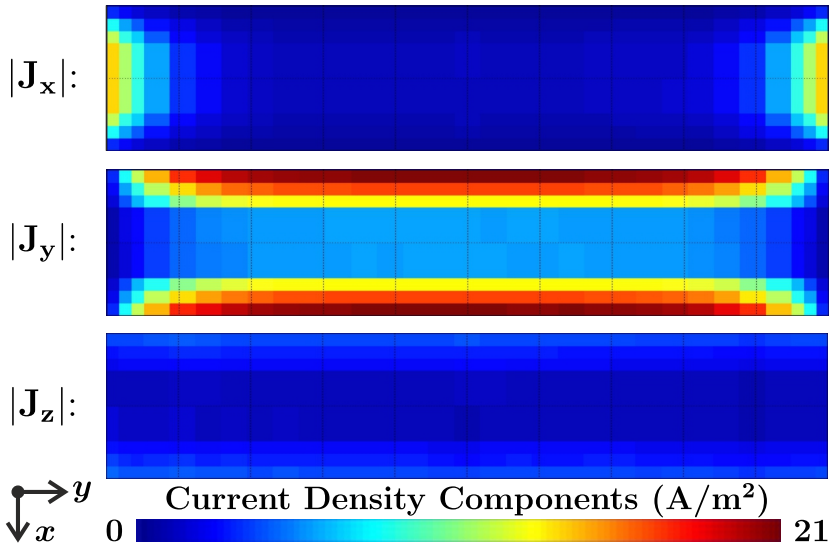

Fig. 4. Current density in the horizontal mid-section of the buried object.

the PEEC approach by comparison with the 2-D FEM solution of a related problem. In this latter approach the buried object is supposed to have an infinite length and $\mathbf{J}$ has a single component in the direction parallel to the transmission line.

Due to a symmetry argument, this solution may be compared with the PEEC solution obtained in the mid-section of the buried object, which is highlighted in Fig. 1(b). A very good agreement between the two solutions is verified. For instance, if the mean current density is computed on a $0.25 \delta \times 0.25 \delta$ patch in the upper corner of the referred midsection, an error lower than $2.15 \%$ is verified between both solutions $\left(\left|\mathbf{J}_{\text {PEEC }}\right|=17.39 \mathrm{~A} / \mathrm{m}^{2},\left|\mathbf{J}_{\text {FEM 2-D }}\right|=17.77 \mathrm{~A} / \mathrm{m}^{2}\right.$, $\delta=$ skin depth $\approx 0.205 \mathrm{~m}$ ).

\section{B. Orthogonal alignment and 3-D FEM validation}

In a second analysis, the object is rotated and positioned with its largest dimension along a direction orthogonal to the transmission line. The resulting configuration can be handled by the PEEC technique as before, but the obtained current density distribution inside the object is no longer comparable with the one issued from a 2-D FEM computation.

The spatial distribution of $\mathbf{J}$ inside the object resulting from the coupling with the three-phase line and computed with the PEEC approach is shown in Fig. 3. Fig. 4 in its turn brings a somewhat more quantitative view of the eddy current loops established inside the object by showing the absolute value of each component of $\mathbf{J}$ in its horizontal mid-section $(z=-0.3 \mathrm{~m})$.

Further insight into the PEEC approach may be obtained by its comparison with an alternative 3-D FEM model in this case. More specifically, an implementation of this method provided by the Flux software package and belonging to the class of $\mathbf{t}-\mathbf{t}_{\mathbf{0}}-\phi$, circuit-coupled formulations [11] [12] was adopted for this purpose.

The 3-D FEM solution of the aforementioned problem is computationally demanding. With this method, the determination of an induced current distribution in the buried object that remains insensitive to additional refinements in the discretization requires a very dense mesh of hexahedra, long computation times and a large memory capacity. 
However, the maximum current density value inside the object may be approximated by an asymptotic value of approximately $21.85 \mathrm{~A} / \mathrm{m}^{2}$. This limiting value is obtained by the 3-D FEM computation of the maximum current density for a series increasingly refined meshes and by extrapolation, as implied by Fig. 5 .

If this value $\left|\mathbf{J}_{\max }\right|=21.85 \mathrm{~A} / \mathrm{m}^{2}$ is adopted as a reference, the maximum current densities computed with the PEEC scheme and the 3-D FEM may be compared. Table I provides this comparison of accuracy between the two procedures when the maximum current density values developed inside the underground object are evaluated with practical and relatively coarse meshes, both containing a similar number of elements $(\approx 5000$ hexahedra $)$. It may be verified that for a given mesh size the PEEC approach is able to provide a more accurate solution than the 3-D FEM.

\section{CONCLUSiOns}

A PEEC approach for the analysis of an inductive coupling problem involving a three-phase line and an underground object was presented. Arbitrary relative positions between the transmission line and the object could be considered, and the method also avoids the discretization of inactive air regions.

The current density distribution obtained provides base data for the study of AC corrosion phenomena [13], and the computed results were validated by comparison with $2-\mathrm{D}$ and 3-D FEM models. Accurate solutions (in the sense provided by a small percent deviation from FEM-computed values) were determined with the use of the PEEC approach.

It should be additionally remarked that the electric potential solution in the domain $\Omega$ resulting from the equivalent circuit interpretation could also be conveniently employed for the evaluation of dangerous induced overvoltages in the PEEC analysis. The use of more complex external networks could also be exploited in the investigation of other practical problems arising in the practice of power engineering, like the occurrence of a simultaneous fault to earth in the threephase line. Future works on numerical aspects like improved integration procedures, system assembly techniques and matrix pre-conditioning could also be envisioned.

Finally, the previously discussed applications considered only the case of a balanced system of three-phase currents

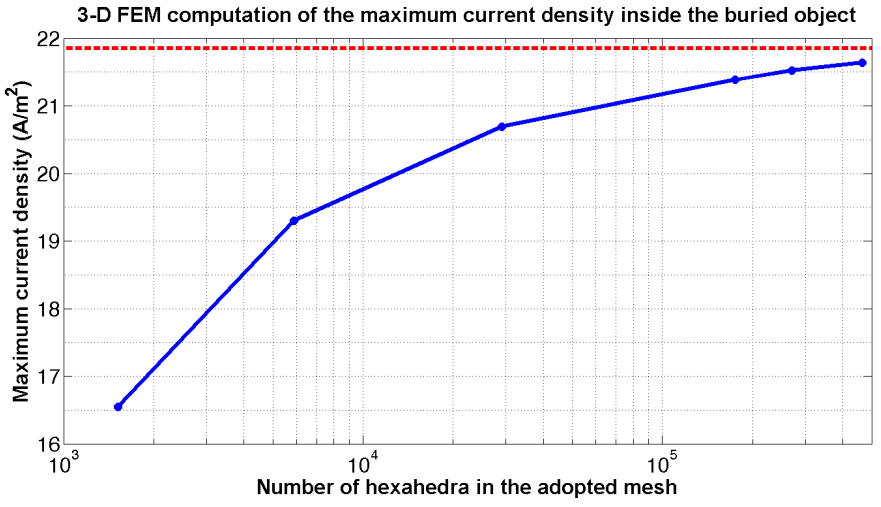

Fig. 5. Determination of the asymptotic value of the maximum current density inside the buried object with the 3D-FEM (orthogonal case).
TABLE I

PEEC AND 3-D FEM COMPARISON (ORTHOGONAL CASE)

\begin{tabular}{cccc}
\hline Method & Mesh size $^{\dagger}$ & $\left|\mathbf{J}_{\max }\right|^{\ddagger}$ & $\%$ Deviation $^{*}$ \\
\hline Reference** & - & 21.85 & 0 \\
3-D FEM & 5880 & 19.30 & 11.67 \\
PEEC & 5040 & 21.20 & 2.97 \\
\hline
\end{tabular}

${ }^{\dagger}$ Number of hexahedra in the discretization.

$\ddagger$ Maximum current density inside buried object $\left(\mathrm{A} / \mathrm{m}^{2}\right)$.

* In comparison with the reference value.

** Extrapolation of the series of 3-D FEM computations (Fig. 5).

flowing in the phase conductors of the transmission line. Further work on this subject also intends to investigate the case of unbalanced operation and the consequent superposition of conductive coupling phenomena, resulting from the flow of zero-sequence current components in the soil.

\section{ACKNOWLEDGEMENT}

The authors would like to acknowledge FAPESP (grants 2011/03450-1 and 2013/21888-0, São Paulo Research Foundation).

\section{REFERENCES}

[1] F. Dawalibi and R. Southey, "Analysis of electrical interference from power lines to gas pipelines. I. computation methods," Power Delivery, IEEE Transactions on, vol. 4, no. 3, pp. 1840-1846, Jul 1989.

[2] G. Christoforidis, D. Labridis, and P. Dokopoulos, "Inductive interference on pipelines buried in multilayer soil due to magnetic fields from nearby faulted power lines," Electromagnetic Compatibility, IEEE Transactions on, vol. 47, no. 2, pp. 254-262, May 2005.

[3] T.-T. Nguyen, G. Meunier, J.-M. Guichon, O. Chadebec, and T.-S Nguyen, "An integral formulation for the computation of 3-D eddy current using facet elements," Magnetics, IEEE Transactions on, vol. 50, no. 2, pp. 549-552, Feb 2014.

[4] A. Bossavit, "Whitney forms: a class of finite elements for threedimensional computations in electromagnetism," Physical Science, Measurement and Instrumentation, Management and Education - Reviews, IEE Proceedings A, vol. 135, no. 8, pp. 493-500, November 1988.

[5] T.-S. Nguyen, J.-M. Guichon, O. Chadebec, G. Meunier, and B. Vincent, "An independent loops search algorithm for solving inductive PEEC large problems," Progress In Electromagnetics Research M, vol. 23, pp. 53-63, 2012.

[6] A. E. Ruehli, "Equivalent circuit models for three-dimensional multiconductor systems," Microwave Theory and Techniques, IEEE Transactions on, vol. 22, no. 3, pp. 216-221, Mar 1974.

[7] T. T. Nguyen, "Méthode PEEC inductive par élément de facette pour la modélisation des régions conductrices volumiques et minces," $\mathrm{Ph}$.D. dissertation, Université de Grenoble, Oct. 2014, in French. [Online]. Available: https://hal.archives-ouvertes.fr/tel-01115779; visited on 28 June 2015.

[8] S. Börm and L. Grasedyck, "Hybrid cross approximation of integral operators," Numerische Mathematik, vol. 101, no. 2, pp. 221-249, 2005. [Online]. Available: http://dx.doi.org/10.1007/s00211-005-0618-1

[9] J. J. LaForest, Transmission Line Reference Book - $345 \mathrm{kV}$ and Above, 2nd ed. Palo Alto, CA: Electric Power Research Institute, 1982.

[10] "Flux 12.0 - Electromagnetic and thermal finite element analysis software package," CEDRAT. [Online]. Available: http://www.cedrat.com/ ; visited on 28 June 2015.

[11] G. Meunier, Y. Le Floch, and C. Guerin, "A nonlinear circuit coupled tt0- phi; formulation for solid conductors," Magnetics, IEEE Transactions on, vol. 39, no. 3, pp. 1729-1732, May 2003.

[12] Y. Le Floch, G. Meunier, C. Guerin, P. Labie, X. Brunotte, and D. Boudaud, "Coupled problem computation of 3-d multiply connected magnetic circuits and electrical circuits," Magnetics, IEEE Transactions on, vol. 39, no. 3, pp. 1725-1728, May 2003.

[13] Evaluation of AC corrosion likelihood of buried pipelines applicable to cathodically protected pipelines, CEN - European Comittee for Standarization. EN 15 280, Sep 2013. 